\title{
Total Synthesis of the Angucylinone Antibiotic (+)-Rubiginone $\mathbf{B}_{\mathbf{2}}{ }^{1}$
}

\author{
Aris Kalogerakis, Ulrich Groth* \\ Fachbereich Chemie, Universität Konstanz, Fach M-720, Universitätsstraße 10, 78457 Konstanz, Germany \\ Fax +49(7531)884155; E-mail: ulrich.groth@uni-konstanz.de
}

\begin{abstract}
A new chiral synthesis of (+)-rubiginone $\mathrm{B}_{2}$ is reported. The intramolecular cobalt-mediated $[2+2+2]$-cycloaddition of a triyne precursor, synthesized from (+)-citronellal, afforded a chiral anthracene, which led after a two-step oxidation to the angucyclinone antibiotic.
\end{abstract}

Key words: angucyclinone antibiotics, benz $[a]$ anthraquinones, natural products, catalysis, cobalt, cycloaddition

The rubiginones (Figure 1), ${ }^{2}$ isolated from the strain of Streptomyces griseorubiginosus, exhibit potential vincristine-induced cytotoxicity against multi-drug-resistant tumor cells. They feature the typical benz $[a]$ anthraquinone structure of the angucyclinone antibiotics, a growing class of secondary metabolites with remarkable biological properties. ${ }^{3}$ While several racemic syntheses of these angucyclinones have been achieved, ${ }^{4}$ only a few asymmetric total syntheses have been reported. ${ }^{5}$ The only known total synthesis of (+)-rubiginone $\mathrm{B}_{2}$ has been reported by Carreño and co-workers based on the Diels-Alder reaction between an enantiopure substituted naphthoquinone and a racemic vinylcyclohexene through the kinetic resolution of the diene. The natural product was synthesized with $80 \%$ ee and $9 \%$ overall yield for the longest sequence. ${ }^{6}$<smiles>COc1cccc2c1C(=O)c1ccc3c(c1C2=O)C(=O)CC(C)C3P</smiles>

rubiginone $A_{2} \quad \mathrm{R}=\mathrm{OH}$

rubiginone $B_{2} R=H$

rubiginone $\mathrm{C}_{2} \mathrm{R}=\mathrm{OCOCH}\left(\mathrm{CH}_{3}\right)_{2}$

Figure 1

We have recently reported a new method ${ }^{1}$ for the construction of the angucyclinone core via an intramolecular cobalt-mediated $[2+2+2]$-cycloaddition of a triyne. ${ }^{7}$ Herein, we describe a new asymmetric access to the angucyclinone antibiotic (+)-rubiginone $B_{2}$ 12. Starting from commercially available $R-(+)$-citronellal $\left([\alpha]_{\mathrm{D}}{ }^{20}+13.0\right.$, neat, $92 \%$ ee $)^{8}$ we have synthesized the chiral diyne 6 (Scheme 1). By employing our previously reported method $^{1}$ we used 6 for the synthesis of the triyne 9, which after cyclization and two-step oxidation led to (+)-rubiginone $\mathrm{B}_{2} \mathbf{1 2}$ (Scheme 2).

Our synthesis began with a Corey-Fuchs olefination ${ }^{9}$ of (+)-citronellal 1. The resulting dibromoalkene $\mathbf{2}$ was used without further purification and converted to the protected alkyne 3 by treatment with $n$-BuLi and then TMS-Cl ${ }^{10}$ (76\% yield, 2 steps). Ozonolysis of $\mathbf{3}\left(\mathrm{Me}_{2} \mathrm{~S}\right.$ workup) afforded 4 in quantitative yield, which was transformed without purification via another Corey-Fuchs olefination into the dibromoalkene $\mathbf{5}$. Treatment of $\mathbf{5}$ with $n$-BuLi and hydrolysis of the resulting lithiated acetylene gave the diyne 6 ( $74 \%$ yield, 3 steps).
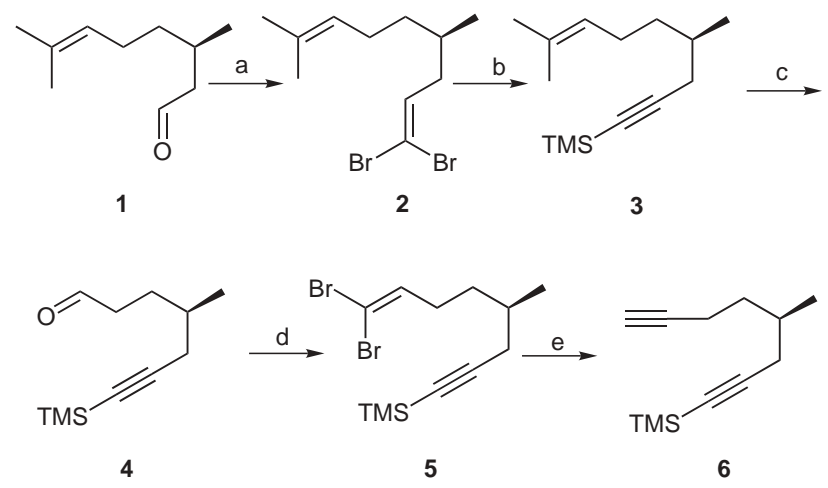

Scheme 1 Reaction Conditions: (a) 2 equiv $\mathrm{CBr}_{4}, 4$ equiv $\mathrm{PPh}_{3}$, $\mathrm{CH}_{2} \mathrm{Cl}_{2}, 0{ }^{\circ} \mathrm{C}, 30 \mathrm{~min}$, then $1,25{ }^{\circ} \mathrm{C}, 2$ h. (b) i. 2 equiv $n$-BuLi, THF, $-80{ }^{\circ} \mathrm{C}, 1 \mathrm{~h}$, ii. 2 equiv TMS-Cl, $-80{ }^{\circ} \mathrm{C}$ to r.t., $18 \mathrm{~h}$ (76\%, 2 steps). (c) i. $\mathrm{O}_{3}, \mathrm{CH}_{2} \mathrm{Cl}_{2},-80{ }^{\circ} \mathrm{C}, 15 \mathrm{~min}$, ii. $\mathrm{Me}_{2} \mathrm{~S}, \mathrm{AcOH},-80{ }^{\circ} \mathrm{C}$ to r.t., 18 h. (d) 2 equiv $\mathrm{CBr}_{4}, 4$ equiv $\mathrm{PPh}_{3}, \mathrm{CH}_{2} \mathrm{Cl}_{2}, 0{ }^{\circ} \mathrm{C}, 30 \mathrm{~min}$, then 4,25 ${ }^{\circ} \mathrm{C}, 2$ h. (e) i. 2 equiv $n$-BuLi, THF, $-80^{\circ} \mathrm{C}, 1$ h, ii. $\mathrm{H}_{2} \mathrm{O},-80{ }^{\circ} \mathrm{C}$ to r.t., 2 h $(74 \%, 3$ steps $)$.

After addition of the lithiated diyne 6 to the benzaldehyde $7^{1}$ and deprotection of the triple bonds of the resulting triyne $\mathbf{8}$ with $\mathrm{NH}_{4} \mathrm{~F},{ }^{11}$ the hydroxy group of the obtained triyne was protected with TBDMSOTf. ${ }^{12}$ Cyclization of 9 with $10 \% \mathrm{CpCo}(\mathrm{CO})_{2}{ }^{13}$ in refluxing toluene under irradiation (tungsten-lamp) gave the anthracene 10 in $74 \%$ yield. By using 5\% $\mathrm{CpCo}(\mathrm{CO})_{2} \mathbf{1 0}$ was obtained in $61 \%$ yield. The use of the expensive $\mathrm{CpCo}$ (ethene) ${ }_{2}{ }^{14}$ for the cyclization (see ref. ${ }^{1}$ ) of the triyne 9 was not necessary. Oxidation with $\left[\mathrm{Ag}(\mathrm{Py})_{2}\right] \mathrm{MnO}_{4}{ }^{15}$ led to the anthraquinone $11(62 \%$ yield), which was converted via photooxidation ${ }^{4 \mathrm{c}}$ to $(+)-$ rubiginone $B_{2} 12$ (67\% yield). ${ }^{16}$ The spectroscopic data of synthetic (+)-rubiginone $\mathrm{B}_{2} \mathbf{1 2}$ coincided with those of the 
natural compound. ${ }^{2}$ The structure of $\mathbf{1 2}$ was proven by two-dimensional NMR experiments (ROESY-, H,HCOSY-spectra, see the H,H-correlations in Figure 2). Additionally, the observed optical rotation value $[\alpha]_{\mathrm{D}}^{25}$ $+71.64\left(c 0.275, \mathrm{CHCl}_{3}, 92 \%\right.$ ee) agreed satisfactory with the reported value $[\alpha]_{\mathrm{D}}{ }^{25}+78.00\left(\mathrm{c} 0.5, \mathrm{CHCl}_{3}\right)$ for the natural product.

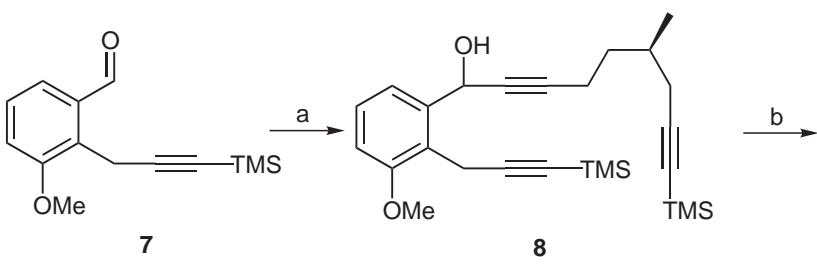<smiles>C#CCc1c(OC)cccc1C(C#CCCC(C)CC#C)OCC</smiles><smiles>COc1cccc2c1C(=O)c1ccc3c(c1C2=O)C(=O)CC(C)C3</smiles>

Scheme 2 Reaction Conditions: (a) $n$-BuLi, 6, THF, $-80{ }^{\circ} \mathrm{C}, 1 \mathrm{~h}$, then $7,-80{ }^{\circ} \mathrm{C}$ to $-30{ }^{\circ} \mathrm{C}, 4 \mathrm{~h}(93 \%)$, (b) i. $\mathrm{NH}_{4} \mathrm{~F}, \mathrm{Bu}_{4} \mathrm{NHSO}_{4}, \mathrm{CH}_{2} \mathrm{Cl}_{2}$, r.t., $48 \mathrm{~h}(96 \%)$, ii. TBDMSOTf, 2,6-lutidine, $\mathrm{CH}_{2} \mathrm{Cl}_{2}, 25{ }^{\circ} \mathrm{C}, 2 \mathrm{~h}$ $(95 \%)(\mathrm{c}) 10 \% \mathrm{CpCo}(\mathrm{CO})_{2}$, toluene, reflux, $\mathrm{h} v, 4 \mathrm{~h}(74 \%)$. (d) 8 equiv $\left[\mathrm{Ag}(\mathrm{Py})_{2}\right] \mathrm{MnO}_{4}, \mathrm{CH}_{2} \mathrm{Cl}_{2}, 25^{\circ} \mathrm{C}, 8 \mathrm{~h}(62 \%)$. (e) $\mathrm{h} v$, air, $\mathrm{CHCl}_{3}, 25^{\circ} \mathrm{C}$, $18 \mathrm{~h}(67 \%)$.

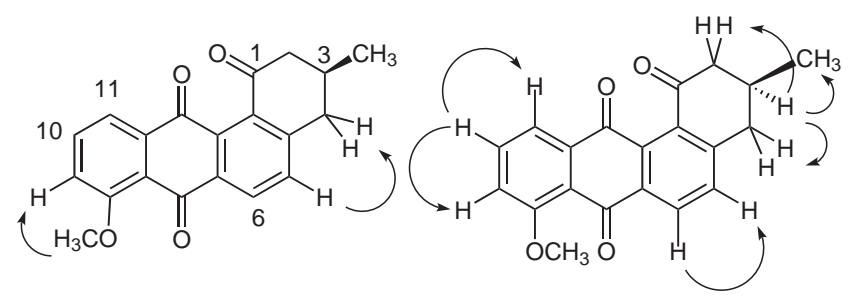

Figure 2 H,H-correlations in ROESY- and H,H-COSY-Spectra

In summary, a new effective chiral synthesis of (+)-rubiginone $\mathrm{B}_{2}$ has been achieved starting from commercially available $R-(+)$-citronellal in 11 steps and $15 \%$ overall yield. Applications to the asymmetric synthesis of other angucyclinone antibiotics using the reported methodology ${ }^{1}$ are currently under investigation.

\section{Acknowledgment}

The authors are grateful to the Fonds der Chemischen Industrie and the EU-Comission, Directorate XII, for financial support. We thank Dr. H. Frauendorf, analytical laboratory of the institute of organic chemistry at the university of Göttingen, for measuring the HRMSanalysis of compound $\mathbf{1 0}$.

\section{References}

(1) Transition metal catalyzed reactions in organic synthesis, part 4. For part 3, see: Kalogerakis, A.; Groth, U. Org. Lett. 2003, 6, 843 .

(2) Oka, M.; Kamei, H.; Hamagishi, Y.; Omita, K.; Miyaki, T.; Konish, M.; Oki, T. J. Antibiot. 1990, 43, 967.

(3) (a) Rohr, J.; Thiericke, R. Nat. Prod. Rep. 1992, 103. (b) Krohn, K.; Rohr, J. Top. Curr. Chem. 1997, 188, 127.

(4) (a) Uemura, M.; Take, K.; Minami, T.; Hayashi, Y. Tetrahedron 1985, 41, 5771. (b) Kraus, G. A.; Wu, Y. Tetrahedron Lett. 1991, 32, 3803. (c) Krohn, K.;

Khanbabaee, K.; Jones, P. G.; Chrapowski, A. Liebigs Ann. Chem. 1994, 471. (d) Krohn, K.; Khanbabaee, K. Liebigs Ann. Chem. 1994, 1109. (e) Krohn, K.; Mcheel, J.; Zukowski, M. Tetrahedron 2000, 56, 4753. (f) Krohn, K.; Böker, N.; Flörke, U.; Freund, C. J. Org. Chem. 1997, 62, 2350. (g) Katsuura, K.; Snieckus, V. Can. J. Chem. 1987, 65, 124. (h) Guingant, A.; Barreto, M. Tetrahedron Lett. 1987, 28, 3107. (i) Patil, M. L.; Borate, H. B.; Ponde, D. E.; Bhawal, B. M.; Deshpande, V. H. Tetrahedron Lett. 1999, 40, 4437. (j) Mal, D.; Roy, H. N. J. Chem. Soc., Perkin Trans 1 1999, 3167. (k) Rozek, T.; Bowie, J. H.; Pyke, S. M.; Skelton, B. W.; White, A. H. J. Chem. Soc., Perkin Trans 1 2001, 1826.

(5) (a) Larsen, D. S.; O’Shea, M. D.; Brooker, S. Chem. Commun. 1996, 203. (b) Caygill, G. B.; Larsen, D. S.; Brooker, S. J. Org. Chem. 2001, 66, 7427. (c) Carreño, M. C.; Ribagorda, M.; Somoza, A.; Urbano, A. Angew. Chem., Int. Ed. 2002, 41, 2755. (d) Boyd, V. A.; Sulikowski, G. A. J. Am. Chem. Soc. 1995, 117, 8472.

(6) (a) Carreño, M. C.; Urbano, A.; Vitta, C. D. Chem. Commun. 1999, 817. (b) Carreño, M. C.; Urbano, A.; Vitta, C. D. J. Org. Chem. 1998, 63, 8320. (c) Hecker, S. J.; Heathcock, C. H. J. Org. Chem. 1985, 50, 5159.

(7) (a) Vollhardt, K. P. C. Acc. Chem. Res. 1977, 10, 1. (b) Vollhardt, K. P. C. Angew. Chem., Int. Ed. Engl. 1984, 23, 539. (c) Lecker, S. H.; Nguyen, N. H.; Vollhardt, K. P. C. J. Am. Chem. Soc. 1986, 108, 856. (d) Dosa, P. I.; Whitener, G. D.; Vollhardt, K. P. C.; Bond, A. D.; Teat, S. J. Org. Lett. 2002, 4, 2075.

(8) (a) Purchased from Fluka. (b) Tietze, L. F.; Kiedrovski, G. V.; Fahlbusch, K.-G.; Voss, E. Org. Synth. 1993, 8, 353. (c) Chapuis, C.; Barthe, M.; Laumer, J.-Y. Helv. Chim. Acta 2001, 84, 230.

(9) Corey, E. J.; Fuchs, P. L. Tetrahedron Lett. 1972, 36, 3769.

(10) Hosokawa, S.; Isobe, M. J. Org. Chem. 1999, 64, 37.

(11) Herold, P. Helv. Chim. Act. 1988, 71, 354

(12) Corey, E. J.; Cho, H.; Ruecker, C.; Hua, D. H. Tetrahedron Lett. 1981, 36, 3455.

(13) Experimental Procedure: A solution of $\mathrm{CpCo}(\mathrm{CO})_{2}(25.00$ $\mathrm{mg}, 0.142 \mathrm{mmol}, 10 \mathrm{~mol} \%$ ) in $2 \mathrm{~mL}$ toluene was added via canula to a solution of triyne $9(0.58 \mathrm{~g}, 1.42 \mathrm{mmol})$ in 120 $\mathrm{mL}$ toluene and the mixture was heated to reflux and irradiated with a tungsten-lamp (osram vitalux $300 \mathrm{~W}$ ) for 4 $h$. The organic phase was then concentrated in vacuo. Chromatography on silica gel $\left(\mathrm{Et}_{2} \mathrm{O}-\right.$ petroleum ether, 1:200) provided anthracene $\mathbf{1 0}(0.29 \mathrm{~g}, 1.051 \mathrm{mmol}, 74 \%$ yield $)$ as a white solid. 
Mp $136-138{ }^{\circ} \mathrm{C} .[\alpha]_{\mathrm{D}}^{22}+86.43\left(c 0.14, \mathrm{CHCl}_{3}\right) . \mathrm{R}_{\mathrm{f}}=0.31$ $\left(\mathrm{Et}_{2} \mathrm{O}\right.$-petroleum ether, 1:100). IR $\left(\mathrm{CCl}_{4}\right): 2935-2810$ [C$\left.\mathrm{H}\left(\mathrm{OCH}_{3}\right)\right] \mathrm{cm}^{-1} .{ }^{1} \mathrm{H}$ NMR $\left(600 \mathrm{MHz}, \mathrm{CDCl}_{3}\right): \delta=1.16(\mathrm{~d}$, $\left.{ }^{3} J=6.6 \mathrm{~Hz}, 3 \mathrm{H}, 3-\mathrm{CH}_{3}\right), 1.58(\mathrm{~m}, 1 \mathrm{H}, \mathrm{H}-2), 2.00(\mathrm{~m}, 1 \mathrm{H}$, H-3), $2.14(\mathrm{~m}, 1 \mathrm{H}, \mathrm{H}-2), 2.60\left(\mathrm{dd},{ }^{2} J=16.5 \mathrm{~Hz},{ }^{3} J=10.5 \mathrm{~Hz}\right.$, $1 \mathrm{H}, \mathrm{H}-4), 2.95\left(\mathrm{dd},{ }^{2} J=16.5 \mathrm{~Hz},{ }^{3} \mathrm{~J}=3.9 \mathrm{~Hz}, 1 \mathrm{H}, \mathrm{H}-4\right), 3.14$ (m, $1 \mathrm{H}, \mathrm{H}-1), 3.40$ (d, $\left.{ }^{2} J=16.5 \mathrm{~Hz}, 1 \mathrm{H}, \mathrm{H}-1\right), 4.09$ (s, $3 \mathrm{H}$, $\left.\mathrm{OCH}_{3}\right), 6.73\left(\mathrm{~d}, J_{\mathrm{o}}=8.0 \mathrm{~Hz}, 1 \mathrm{H}, \mathrm{H}-9\right), 7.18\left(\mathrm{~d}, J_{\mathrm{o}}=8.6 \mathrm{~Hz}\right.$, $1 \mathrm{H}, \mathrm{H}-5), 7.37\left(\mathrm{t}, J_{\mathrm{o}}=8.0 \mathrm{~Hz}, 1 \mathrm{H}, \mathrm{H}-10\right), 7.62\left(\mathrm{~d}, J_{\mathrm{o}}=8.0\right.$ $\mathrm{Hz}, 1 \mathrm{H}, \mathrm{H}-11), 7.82$ (d, $\left.J_{\mathrm{o}}=8.6 \mathrm{~Hz}, 1 \mathrm{H}, \mathrm{H}-6\right), 8.44(\mathrm{~s}, 1 \mathrm{H}$, $\mathrm{H}-12), 8.78$ (s, $1 \mathrm{H}, \mathrm{H}-7) .{ }^{13} \mathrm{C} \mathrm{NMR}\left(100 \mathrm{MHz}, \mathrm{CDCl}_{3}\right): \delta=$ $21.79\left(3-\mathrm{CH}_{3}\right), 25.83(\mathrm{C}-1), 28.88(\mathrm{C}-3), 31.35(\mathrm{C}-2), 39.14$ $(\mathrm{C}-4), 55.45\left(\mathrm{OCH}_{3}\right), 101.34(\mathrm{C}-9), 120.75(\mathrm{C}-12), 120.83$ (C-11), 121.35 (C-7), 125.06 (C-10), 126.58 (C-6), 128.05 (C-5), 124.09, 130.34, 131.42, 132.64, 133.57, 155.38 $\left(\mathrm{C}_{\text {quart }}\right.$-arom. $)$. EI-MS $(70 \mathrm{eV}): \mathrm{m} / \mathrm{z}=276\left(100 \%, \mathrm{M}^{+}\right), 261$ $\left(15 \%, \mathrm{M}^{+}-\mathrm{CH}_{3}\right), 246\left(7 \%, 261-\mathrm{CH}_{3}\right), 233(77 \%, 261-$ $\mathrm{C}_{2} \mathrm{H}_{4}$ ). HRMS: calcd 276.1514 for $\mathrm{C}_{20} \mathrm{H}_{20} \mathrm{O}$, found 276.1508

(14) (a) Jonas, K.; Deffense, E.; Habermann, D. Angew. Chem., Int. Ed. Engl. 1983, 22, 716. (b) Cammack, J. K.; Jalisatgi, S.; Matzger, A. J.; Négron, A.; Vollhardt, K. P. C. J. Org. Chem. 1996, 61, 4798.

(15) (a) Tius, M. A.; Galeno, J. G.; Gu, X.; Zaid, J. H. J. Am. Chem. Soc. 1991, 113, 5775. (b) Firouzabadi, H.; Vessel, B.; Naderi, M. Tetrahedron Lett. 1982, 23, 1847.
(16) Experimental Procedure: Anthraquinone 11 (60.00 mg, $0.195 \mathrm{mmol}$ ) was dissolved in $5 \mathrm{~mL} \mathrm{CHCl}_{3}$ and irradiated with a tungsten-lamp (osram vitalux $300 \mathrm{~W}$ ) over $18 \mathrm{~h}$. The organic layer was then removed in vacuo. Chromatography on silica gel (petroleum ether- $\mathrm{Et}_{2} \mathrm{O}, 1: 2$ ) provided (+)rubiginone $\mathrm{B}_{2} 12(42.00 \mathrm{mg}, 0.131 \mathrm{mmol}, 67 \%$ yield $)$ as a yellow solid. $\mathrm{Mp}>262^{\circ} \mathrm{C}$ (dec.). $[\alpha]_{\mathrm{D}}{ }^{25}+71.64$ (c $0.275, \mathrm{CHCl}_{3}$, recrystallized from petroleum ether- $\left.\mathrm{CHCl}_{3}, 2: 1\right) . \mathrm{R}_{\mathrm{f}}=0.14$ $\left(\mathrm{Et}_{2} \mathrm{O}\right.$-petroleum ether, 1:4). IR $\left(\mathrm{CCl}_{4}\right): 1673,1677,1708$ $(\mathrm{C}=\mathrm{O}) \mathrm{cm}^{-1} \cdot{ }^{1} \mathrm{H} \mathrm{NMR}\left(400 \mathrm{MHz}, \mathrm{CDCl}_{3}\right): \delta=1.20\left(\mathrm{~d},{ }^{3} \mathrm{~J}=\right.$ $\left.6.4 \mathrm{~Hz}, 3 \mathrm{H}, 3-\mathrm{CH}_{3}\right), 2.45(\mathrm{~m}, 1 \mathrm{H}, \mathrm{H}-3), 2.55\left(\mathrm{dd},{ }^{2} \mathrm{~J}=15.6\right.$ $\left.\mathrm{Hz},{ }^{3} J=11.0 \mathrm{~Hz}, 1 \mathrm{H}, \mathrm{H}-2\right), 2.67\left(\mathrm{dd},{ }^{2} J=16.4 \mathrm{~Hz},{ }^{3} J=11.0\right.$ $\mathrm{Hz}, 1 \mathrm{H}, \mathrm{H}-2), 2.98$ (m, $2 \mathrm{H}, \mathrm{H}-1$ and H-4), 4.04 (s, $3 \mathrm{H}$, $\left.\mathrm{OCH}_{3}\right), 7.24\left(\mathrm{~d}, J_{\mathrm{o}}=8.2 \mathrm{~Hz}, 1 \mathrm{H}, \mathrm{H}-9\right), 7.45\left(\mathrm{~d}, J_{\mathrm{o}}=8.0 \mathrm{~Hz}\right.$, $1 \mathrm{H}, \mathrm{H}-5), 7.65\left(\mathrm{t}, J_{\mathrm{o}}=8.2 \mathrm{~Hz}, J_{\mathrm{o}}=7.8 \mathrm{~Hz}, 1 \mathrm{H}, \mathrm{H}-10\right), 7.71$ $\left(\mathrm{d}, J_{\mathrm{o}}=7.8 \mathrm{~Hz}, 1 \mathrm{H}, \mathrm{H}-11\right), 8.20\left(\mathrm{~d}, J_{\mathrm{o}}=8.0 \mathrm{~Hz}, 1 \mathrm{H}, \mathrm{H}-6\right)$. ${ }^{13} \mathrm{C}$ NMR $\left(150 \mathrm{MHz}, \mathrm{CDCl}_{3}\right): \delta=21.43\left(3-\mathrm{CH}_{3}\right), 30.82$ (C-3), 38.33 (C-4), $47.55(\mathrm{C}-2), 56.50\left(\mathrm{OCH}_{3}\right), 117.15$ (C-9), 119.68 (C-11), 129.60 (C-6), 132.99 (C-5), 135.34 (C-10), 120.56, 134.98, 135.07, 137.67, 149.13, 159.81 $\left(\mathrm{C}_{\text {quart }}\right.$-arom. $), 181.59,184.51,198.89(\mathrm{C}=\mathrm{O})$. EI-MS (70 $\mathrm{eV}): m / z=320\left(100 \%, \mathrm{M}^{+}\right), 305\left(15 \%, \mathrm{M}^{+}-\mathrm{CH}_{3}\right), 292$ $\left(35 \%, \mathrm{M}^{+}-\mathrm{CO}\right), 291\left(15 \%, \mathrm{M}^{+}-\mathrm{HCO}\right), 261(27 \%, 292-$ $\left.\mathrm{OCH}_{3}\right), 233(24 \%, 261-\mathrm{CO})$. 